\title{
IL CICLO CLIMATICO DI 8 ANNI E SUA REALTA' FISICA
}

\author{
Silvio Polli
}

1. Premesse. - L'esame delle rappresentazioni grafiche di analisi periodali e armoniche di lunghe serie climatiche mette in vista l'esistenza di alcune fluttuazioni componenti che si distinguono per la loro ampiezza e persistenza. Tra queste figurano i noti cicli di anni 35 (Brückner) $\left({ }^{6}\right)$; 16 (Wagner) $\left(^{34}\right) ; 11,15$ (solare) $\left({ }^{27}\right)$ e 5,6 (Polli) $\left({ }^{-6}\right)$.

In questa nota si determineranno: l'esistenza, i caratteri e la realtà fisica di una oscillazione climatica avente periodo di 8 anni. Essa appare in tutte le analisi periodali finora eseguite. Rispetto alle altre componenti è una delle più persistenti, regolari e ampie. Nelle analisi di serie solari è la più intensa dopo quella undecennale. In generale la sua ampiezza è dello stesso ordine di quella dei cicli di 11 e 5,6 anni.

2. Criterio di realtà fisica. - Il problema che immediatamente si impone nello studio di una oscillazione di carattere geofisico ricavata mediante analisi matematica è quello della sua realtà fisica. I vari criteri proposti si possono dividere in due gruppi: il primo basato su procedimenti di probabilità statistica, come il criterio dell'espettanza di Schuster-Stumpff $\left({ }^{29}\right)$; il secondo su osservazioni empiriche intuitive, come quello del Marvin ( ${ }^{17}$ ).

Il criterio di Schuster-Stumpff, legato a leggi matematiche, male si adatta ad operare su fluttuazioni così variabili ed indefinibili quali sono le oscillazioni degli elementi climatici, e pertanto, per poter comprendere il significato dei risultati a cui esso porta, occorre tener molto conto dell'elasticità della relazione. La rigidezza matematica dell'analisi aggiunta a quella del criterio conducono spesso a risultati contrari a fatti evidenti.

Quello di Marvin, che considera aventi realtà fisica quelle componenti che emergono da un gran numero di analisi, si basa su un buon argomento pratico; per essere applicato con uniformità abbisogna di una definizione più particolareggiata, occorre cioè liberare il concet to fondamentale da influenze personali, in modo che la sua applicazione riesca oggettiva il più possibile. 
In questo lavoro si è seguito il criterio che ora si esporrà, criterio che ha dato risultati di indubbio valore nelle ricerche precedenti $\left(^{26}\right)$.

L'esistenza reale di un ciclo geofisico (in particolare climatico! puó ritenersi praticamente dimostrata quando siano soddisfatte le seguenti condizioni: 1) si presenti in tutto l'intervallo considerato di una stessa successione di valori; 2) si presenti in tutte le serie dei diversi elementi geofisici (climatici) di uno stesso sito; 3) si presenti in tutti i posti della terra; 4/ abbia ampiezza notevole o sufficiente perché i suoi caratteri siano chiaramente determinabili; 5 ) abbia periodo costante o quasi; 6) abbia fase costante o quasi; 7) gli eventuali caratteri particolari (notevole sviluppo o smorzamento) siano comuni a tutti gli elementi e a tutti i posti.

$\dot{E}$ evidente che tali condizioni non saranno sempre rigorosamente sorddisfatte. Errori di osservazione, nel senso più generale, alterano l'andamento originale del fenomeno e pertanto modificano la forma dell'oscillazione componente. Praticamente, per poter considerare il ciclo realmente esistente, basterà che esso presenti caratteri molto prossimi a quelli corrispondenti alle condizioni enunciate.

3. Procedimento. - Sono state esaminate 45 analisi periodali complete, cioè quasi tutte quelle sinora disponibili, una decina di analisi armoniche non tutte complete e numerose ricerche eseguite con altri metodi. Le serie analizzate riguardano elementi climatici, geofisici e astronomici (numeri di Wolf delle macchie solari e diametri solari).

Si è trovato che fra le poche fluttazioni componenti che risaltano per ampiezza, persistenza e regolarità, figura una con periodo medio di 8 anni. Essa si presenta sempre, la sua ampiezza sorpassa talvolta quella di ogni altra componente, spesso è maggiore di quella del ciclo undecennale, quasi sempre supera in ampiezza i cicli di Wagner e di Brückner, è di poco inferiore a quella del ciclo di 5,6 anni; raramente si annulla. E qualche volta sorpassata dalle componenti di 4 anni e da quelle di periodo più hreve, ma solo per intervalli molto limitati. Non ba generalmente la stessa fase in elementi climatici e in regioni diverse, si ba invece concordanza per gli elementi di una medesima regione. Si osservano corrispondenze di carattere molto generale; come per esempio le perturbazioni e gli smorzamenti intorno agli anni 1890 e 1905, irregolarità osservabili anche in altri cicli climatici. L'esistenza del ciclo si può grossolanamente osservare anche dopo avere sottoposto la serie di dati a semplici operazioni di perequazione. 
4. Risultati di analisi. - Presentiamo l'elenco degli elementi climatici e geofisici analizzati, e dei siti considerati. Per ciascuno di essi sono generalmente indicati: la posizione geografica, l'intervallo di anni considerato, il periodo medio in anni e decimi, l'ampiezza massima dell'onda completa osservata nell'intervallo, $i$ caratteri più notevoli, l'autore dell'analisi e la pubhlicazione riguardante la stessa o la serie considerata. Quando il metodo di esame non è specificato, si intende quello dell'analisi periodale secondo il procedimento Vercelli ${ }^{33}$ ).

5. Pressione atmosferica. - Venezia. $45^{\circ} 26^{\prime} \mathrm{N}, 12^{\circ} 18^{\prime} \mathrm{E}, \mathrm{h}=21$ m; 1876-1941. Periodo medio: 8 anni; ampiezza massima $1,5 \mathrm{mb}$. É fra le componenti più ampie, è maggiore di quelle di 11 e 5,6 anni.

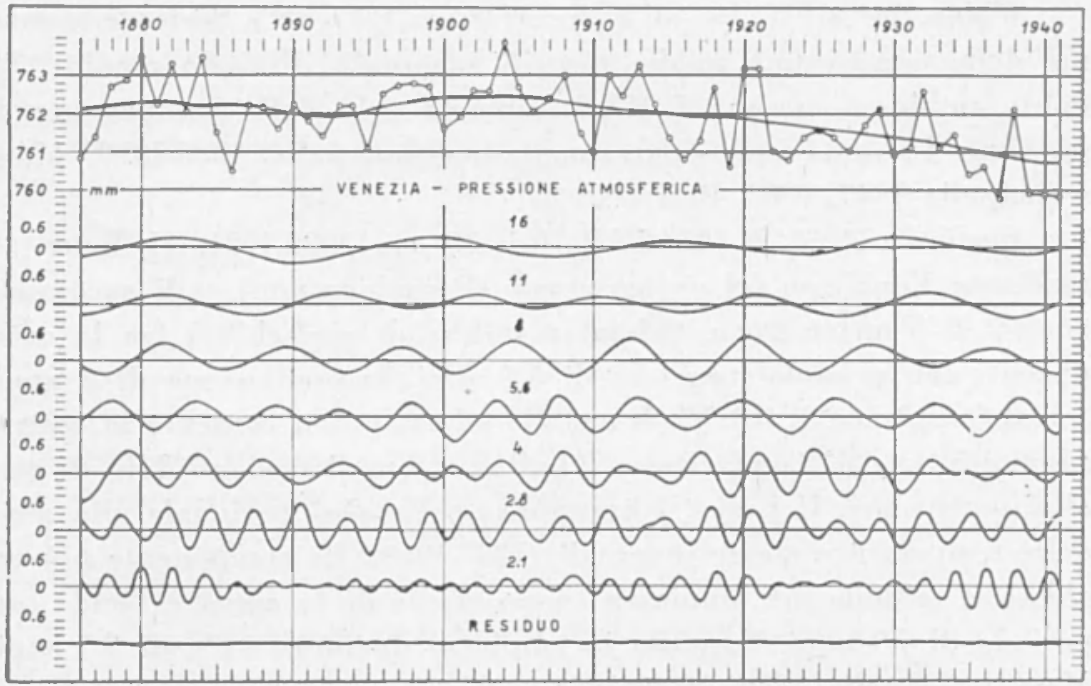

Fig. 1 - Analisi periodale della serie delle pressioni atmosferiche medie di Venezia

Si presenta regolare con sviluppo più marcato negli anni 1905-20. Mass. principali : 1882, 1912, 1929. Polli ( $\left.{ }^{31}\right)$.

Roma. $41^{0} 54^{\prime} \mathrm{N}, 12^{\circ} 29^{\prime} \mathrm{E}, \mathrm{h}=50 \mathrm{~m}$; 1858-1910. Periodo medio: 8 anni; ampiezza massima $1,2 \mathrm{mb}$; supera notevolmente quella underennale, è dello stesso ordine dell'onda di 5,6 anni. Si presenta regolare e uniforme. Mass. principali: 1873, 1889. Polli (11).

Regione alpina. (Basilea, Zurigo, Ginevra, Lugano). Il Maurer $\left({ }^{18}\right.$ ) esaminando la serie delle somme degli scostamenti invernali di varie località alpine nel periodo 1836-1917, trova una preponderante, mar- 
catissima e regolare fnItuazione di 3 anni, rilevabile immediatamente anche senza perequazioni. I massimi cadono negli inverni 1865-66, 1873-74, 1881-82, 1889-90, 1897-98, 1904-05, 1912-13.

6. TEMPERATURA DELL'ARIA. - Stoccolma. $59^{\circ} 21^{\prime} \mathrm{N}, 18^{\prime} 3^{\prime} \mathrm{E}, \mathrm{h}=44$ $\mathrm{m}$; 1777-1927. Serie delle somme delle temperature medie dei 3 mesi invernali. Periodo medio: 8,3 anni. É la più ampia di tutte le componenti; presenta massimi sviluppi negli intervalli 1820-50, 1870-1900, risulta dehole nei periodi 1790-1805, 1855-70. Mass. principali: 1829 , 1886. Buli $\left({ }^{7}\right)$. Il Woeikof $\left.{ }^{(35}\right)$ esaminando, mediante semplici accostamenti, la successione delle temperature invernali dal 1756 al 1906, trova un notevole periodo di 7,9 anni, particolarmente marcato negli ultimi 90 anni.

Wilno. $54^{0} 41^{\prime} \mathrm{N}, 25^{\circ} 18^{\prime} \mathrm{E}, \mathrm{h}=148 \mathrm{~m}$; 1837-1915. Serie delle somme delle temperature medie mensili invernali. Periodo medio: 7,9 anni; ampiezza masima $5{ }^{\circ} \mathrm{C}$, è superata solo dalla componente di 16 anni. Presenta uno smorzamento nell'intervallo 1885-1900. Mass. principali : 1847 , 1887. Buli ( $\left.{ }^{7}\right)$.

Berlino. $52^{\circ} 33^{\prime} \mathrm{N}, 13^{0} 2 \mathrm{l}^{\prime} \mathrm{E}, \mathrm{h}=35 \mathrm{~m}$; 1822-1921. Il Baur ( $\left.{ }^{4}\right)$ mediante l'impiego del ciclogramma (Periodogramm) e di successive analisi di Fourier trova, prima in ordine di probabilità fra le componenti con periodo maggiore di 5,6 anni, la oscillazione di 8 anni, con un'ampiezza di $0,3{ }^{\circ} \mathrm{C}$. Il metodo adottato non consente la costruzione effettiva dell'onda stessa e pertanto non si possono dare di essa altri particolari. Il Buli $\left({ }^{7}\right)$ ha applicato l'analisi periodale alla serie delle temperature medie invernali (1827-1920). La componente più regolare, e seconda per ampiezza (dopo quella di 16 anni), è l'onda con periodo di 7,9 anni. Sorpassa in ampiezza quelle di 11 e di 5,6 anni. Mass. principali: 1846, 1890, 1901.

De Bilt. 52" 6' N, $5^{0} 11^{\prime} \mathrm{E}, \mathrm{h}=3 \mathrm{~m} ; 1$ 1706-1944. Periodo medio: 8 anni; ampiezza massima $1,1{ }^{\circ} \mathrm{C}$; leggermente inferiore a quella del ciclo di 11 anni. Si presenta abbastanza regolare e uniforme, ha massimo sviluppo nell'intervallo 1820-40, subisce smorzamenti intorno agli anni 1740, 1800, 1885. Massimi principali: 1729, 1773, 1834, 1920. Polli ( $\left.{ }^{14}\right)$.

Lipsia. $51^{0} 21^{\prime} \mathrm{N}, 12^{\prime} 22^{\prime} \mathrm{E}, \mathrm{h}=110 \mathrm{~m} ; 1$ 1831-1935. Periodo medio: 8 anni. Ampiezza massima $1,2{ }^{\circ} \mathrm{C}$. E la più ampia fra quelle di periodo superiore a 5, 6 anni. Si mantiene regolare, presenta solo uno smorzamento intorno al 1897. Massimi principali : 1875, 1912. Polli $\left({ }^{20}\right)$.

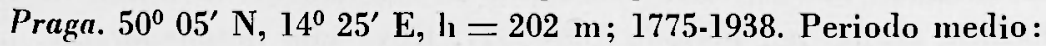


8 anni. Ampiezza massima $1,0{ }^{\circ} \mathrm{C}$, di poco inferiore a quelle del ciclo di 11 e di 5,6 anni. Ha massimo sviluppo nell'intervallo 1820-1840; appare smorzata intorno agli anni 1850 e 1895. Massimi principali: 1834, 1876. Polli ( $\left.{ }^{12}\right)$.

Vienna. $48^{0} 15^{\prime} \mathrm{N}, 16^{0} 22^{\prime} \mathrm{E}, \mathrm{l}=203 \mathrm{~m} ;$ 1827-1927. Serie delle somme delle temperature medie dei mesi invernali. Periodo medio: 8 anni. Ampiezza massima $4,5{ }^{\circ} \mathrm{C}$, è al secondo posto dopo quella di 16 anni, supera pertanto le onde di 11 e di 5,6 anni. Si indebolisce dopo il 1890. Massimi principali: $1848,1871,1888$. Buli $\left({ }^{7}\right)$.

Gran San Bernardo. $45^{\circ} 52^{\prime} \mathrm{N}, 7^{0} 10^{\prime} \mathrm{E}, \mathrm{b}=2467 \mathrm{~m}$; 1818-1931. Temperature medie estive. Periodo medio: 7,6 anni. Ampiezza media $0,26{ }^{\circ} \mathrm{C}$. Presenta notevoli fluttuazioni di ampiezza. Lovera $\left({ }^{16}\right)$.

Trieste. $45^{\circ} 39^{\prime} \mathrm{N}, 13^{\circ} 45^{\prime} \mathrm{E}, \mathrm{h}=11 \mathrm{~m}$; 1841-1940. Periodo medio: 8 anni. Ampiezza massima $0.5{ }^{\circ} \mathrm{C}$. E ampia come la componente

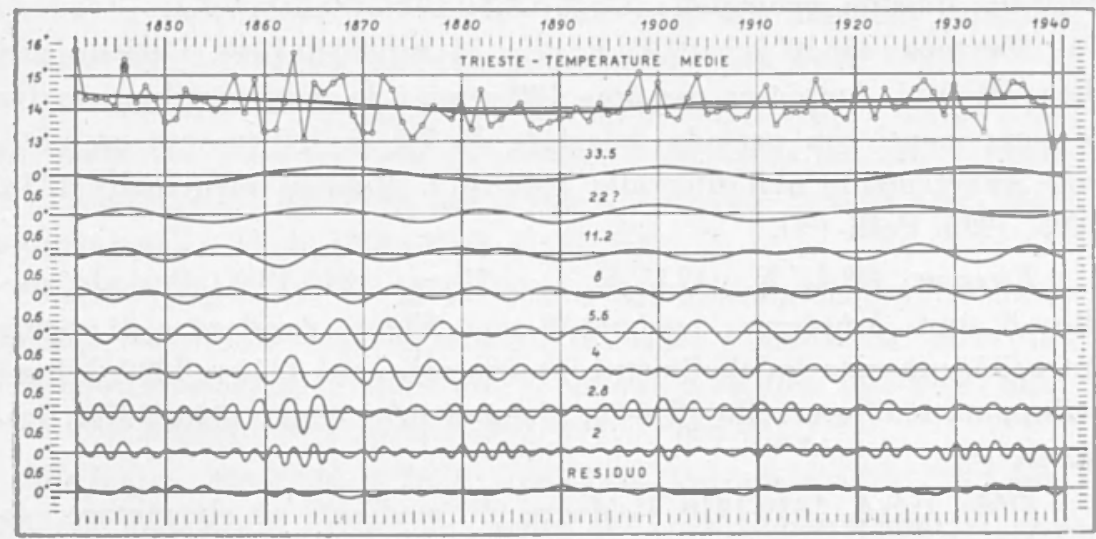

Fig. 2 Analisi periodale della serie delle temperature medie di Trieste

undecennale. Si sviluppa regolare con due lievi indebolimenti intorno agli anni 1890 e 1910 . Polli $\left({ }^{23}\right)$.

Torino. $45^{\circ} \mathrm{l}^{\prime} \mathrm{N}, 7^{0} 39^{\prime} \mathrm{E}, \mathrm{l}=42 \mathrm{~m}$ : 1753-1940. Periodo medio: 7,8 anni. Ampiezza massima $1{ }^{\circ} \mathrm{C}$. Presenta andamento vario con massimo sviluppo nell'intervallo 1805-1835, si smorza intorno agli anni 1798, 1875; in quest'ultima epoca subisce una variazione di fase. Massimi principali: 1780,1818 . Lovera $\left({ }^{15}\right)$.

Roma, $41^{\circ} 54^{\prime} \mathrm{N}, 12^{\circ} 29^{\prime} \mathrm{E}, \mathrm{h}=50 \mathrm{~m}$; 1860-1920. Periodo medio: 8 anni. Ampiezza massima $3,5{ }^{\circ} \mathrm{C}$. Maggiore di quella del ciclo undecennale, si indebolisce nell'intervallo 1880-90. Massimi principali : 1864, 1904. Sella $\left({ }^{32}\right)$. 
7. Precipitazioni totali annue. - Gran San Bernardo (Osservatorio). $45^{\circ} 52^{\prime} \mathrm{N}, 7^{\prime \prime} 10^{\prime} \mathrm{E}, \mathrm{h}=2467 \mathrm{~m}$; 1818-1931. Precipitazioni nevose. Periodo medio: 8,2 anni. Ampiezza media $206 \mathrm{~cm}$, maggiore di quella della componente undecennale che è di $114 \mathrm{~cm}$. Presenta notevole sviluppo sino al 1895 circa. Lovera $\left({ }^{16}\right)$.

Trieste. $45^{\circ} 39^{\prime} \mathrm{N}, 13^{0} 45^{\prime} \mathrm{E}, \mathrm{h}=18 \mathrm{~m}$; 1841-1940. Periodo medio: 8 anni. Ampiezza massima $200 \mathrm{~mm}$; è la meglio sviluppata fra le componenti aventi periodo maggiore di 4 anni, solamente intorno al 1900 presenta un notevole smorzamento con cambiamento di fase. Massimi principali: 1862, 1924. Polli ${ }^{23}$ ).

Padova. $45^{\circ} 24^{\prime} \mathrm{N}, 11^{0} 50^{\prime} \mathrm{E}, \mathrm{h}=15 \mathrm{~m} ; 1727-1940$. Periodo medio: 8 anni. Ampiezza massima $230 \mathrm{~mm}$; è la più ampia fra le componenti aventi periodo maggiore di 5,6 anni. Si smorza intorno agli anni 1754, 1810, 1887. Presenta notevole sviluppo negli intervalli 1760-95, 1840-60, 1920-40. Massimi principali : 1772, 1885, 1927. Polli ( ${ }^{22}$ ).

Mantova. $45^{\circ} 10^{\prime} \mathrm{N}, 10^{\circ} 45^{\prime} \mathrm{E}, \mathrm{h}=54 \mathrm{in} ; 1840-1940$. Periodo medio: 7,9 anni. Ampiezza massima $180 \mathrm{~mm}$; è la meglio sviluppata fra le componenti con periodo maggiore di 5,6 anni. Presenta un notevole smorzamento nell'intervallo 1865-1877. Massimi principali: 1853, 1895, 1925. Polli $\left({ }^{25}\right)$.

Firenze. $43^{\circ} 47^{\prime} \mathrm{N}, 11^{0} 15^{\prime} \mathrm{E}, \mathrm{h}=75 \mathrm{~m} ;$ 1813-1918. Periodo medio: 8 anni. Ampiezza massima 18 mm. Figura regolare e di media intensità, presenta indebolimenti intorno agli anni 1830 e 1890 , è bene sviluppata nell'intervallo 1840-70. Massimi principali: 1846, 1878. Bramanti (").

Ohio, U.S.A. 1839-19]0. Il Moore (19) mediante un elementare esame della successione trova un periodo di 8 anni con un'ampiezza media di $105 \mathrm{~mm}$.

Bogota. $4^{0} 35^{\prime} \mathrm{N}, 74^{0} 14^{\prime} \mathrm{W}, \mathrm{h}=2660 \mathrm{~m} ; 1866-1925$. Periodo medio: 8,1 anni. Ampiezza massima $210 \mathrm{~mm}$. Si smorza e cambia fase nell'intervallo 1892-1896. Massimi principali: 1878, 1899. Polli ( $\left.{ }^{13}\right)$.

Rio de Janeiro. $22^{0} 54^{\prime} \mathrm{S}, 43^{\circ} 10^{\prime} \mathrm{W}, \mathrm{h}=60 \mathrm{~m} ;$ 1851-1925. Periodo medio 7,9 anni. Ampiezza massima $380 \mathrm{~mm}$. È la più ampia delle componenti; presenta massimo sviluppo nell'intervallo 1870-90; si smorza e cambia fase intorno all'anno 1908. Massimi principali: 1874, 1882. Polli (13).

Buenos Aires. $34^{0} 37^{\prime} \mathrm{S}, 58^{0} 22^{\prime} \mathrm{W}, \mathrm{h}=25 \mathrm{~m} ; 1856-1900$. Periodo medio: 7,7 anni. Ampiezza massima $180 \mathrm{~mm}$; si smorza intorno al 1890 . Massimi principali: 1870. Polli ( $\left.{ }^{9}\right)$. 
8. Livelli medi maRini. - Le altezze dei livelli medi marini sono molto sensibili alle lente fluttuazioni climatiche. Variazioni anche minime della temperatura, precipitazione e umidità, difficilmente apprezzahili con dirette misure strumentali, si rendono evidenti e misurabili nelle corrispondenti variazioni dei livelli medi. Sono state analizzate pertanto numerose serie di valori medi annui, qualcuna anche ultrasecolare, dedotte da registrazioni mareografiche regolari e continue.

Lyökki. $60^{\circ} 51^{\prime} \mathrm{N}, 21^{\circ} 11^{\prime} \mathrm{E}, 1858-1936$. Periodo medio: 7,9 anni; ampiezza massima $7 \mathrm{~cm}$. Fra le componenti di periodo superiore agli 5,6 anni è la più ampia. Assume massimo sviluppo dal 1905 al 1915; si smorza e cambia fase nell'intervallo 1880-1895. Massimi principali: 1866, 1913. Polli $\left({ }^{3}\right)$.

Söderskar. $60^{\circ} 7^{\prime} \mathrm{N}, 25^{\circ} 25^{\prime} \mathrm{E}$; 1866-1936. Periodo medio: 8,1 anni; ampiezza massima $8 \mathrm{~cm}$. E la più ampia fra le componenti a lungo periodo. Si indebolisce intorno al 1885 , presenta massimo sviluppo nell'intervallo 1895-1915. Massima principale: 1904. Polli ( $\left.{ }^{3}\right)$.

Suinemïnde. $53^{0} 56^{\prime} \mathrm{N}, 14^{0} 17^{\prime} \mathrm{E}$; 1811-1936. Periodo medio: 8,1 anni; ampiezza massima $6 \mathrm{~cm}$. Si presenta abbastanza uniforme tranne che nell'intervallo 1880-1900, nel quale si smorza e cambia fase. Massimi principali : 1819, 1844, 1905. Polli ( $\left.{ }^{3}\right)$.

Wismar. 53' $54^{\prime} \mathrm{N}, 11^{0} 28^{\prime} \mathrm{E}$; 1849-1936. Periodo medio: 8 anni: ampiezza massima $5 \mathrm{~cm}$, leggermente inferiore a quella del ciclo undecennale; è abbastanza uniforme, si smorza e cambia fase negli anni 1880-1885. Massimi principali: 1860, 1930. Polli $\left({ }^{3}\right)$.

Harlingen. $53^{\circ} 10^{\prime} \mathrm{N}, 5^{0} 25^{\prime} \mathrm{E}$; 1865-1936. Periodo medio: 7,8 anni; ampiezza massima $4 \mathrm{~cm}$, come quella del ciclo undecennale; si presenta uniforme con un leggero smorzamento intorno all'anno 1895. Massimi principali : 1875, 1905. Polli ( $\left.{ }^{24}\right)$.

Massluis. $51^{0} 55^{\prime} \mathrm{N}, 4^{0} 15^{\prime} \mathrm{E}$; 1848-1936. Periodo medio: 8 anni; ampiezza massima $6 \mathrm{~cm}$; è la più ampia fra le componenti aventi periodo maggiore 5,6 anni. E ben sviluppata nell'intervallo $1855-80$; si smorza e cambia fase negli anni $1885-95$ e 1908-15. Massimi principali : 1861, 1877, 1924. Polli ( $\left.{ }^{24}\right)$.

Vlissingen. $51^{0} 27^{\prime} \mathrm{N}, 3^{0} 36^{\prime} \mathrm{E}$; 1862-1936. Periodo medio: 8 anni; ampiezza massima $5 \mathrm{~cm}$. Figura bene sviluppata sino al 1887, smorzata dal 1888 al 1898 , meno ampia successivamente. Massimi principali: 1876, 1905. Polli (24).

Trieste. $45^{\circ} 39^{\prime} \mathrm{N}, 13^{\circ} 46^{\prime} \mathrm{E}$; 1890-1942. Periodo medio: 7,5 anni; ampiezza massima $3 \mathrm{~cm}$, corrispondente a c. $2 / 3$ di quella del ciclo 
undecennale. Si smorza e cambia fase nell'intervallo 1926-1931. Massimi principali: 1909, 1931. Polli $\left({ }^{28}\right)$.

Venezia. $45^{\circ} 26^{\prime} \mathrm{N}, 12^{\prime \prime} 20^{\prime} \mathrm{E}$; 1872-1941. Periodo medio: 7,8 anni; ampiezza massima $4 \mathrm{~cm}$, come la componente di 11 anni. Si presenta bene sviluppata e regolare tranne un breve indeloolimento intorno all'anno 1900. Mass. principali: 1886, 1917. Polli $\left({ }^{28}\right)$.

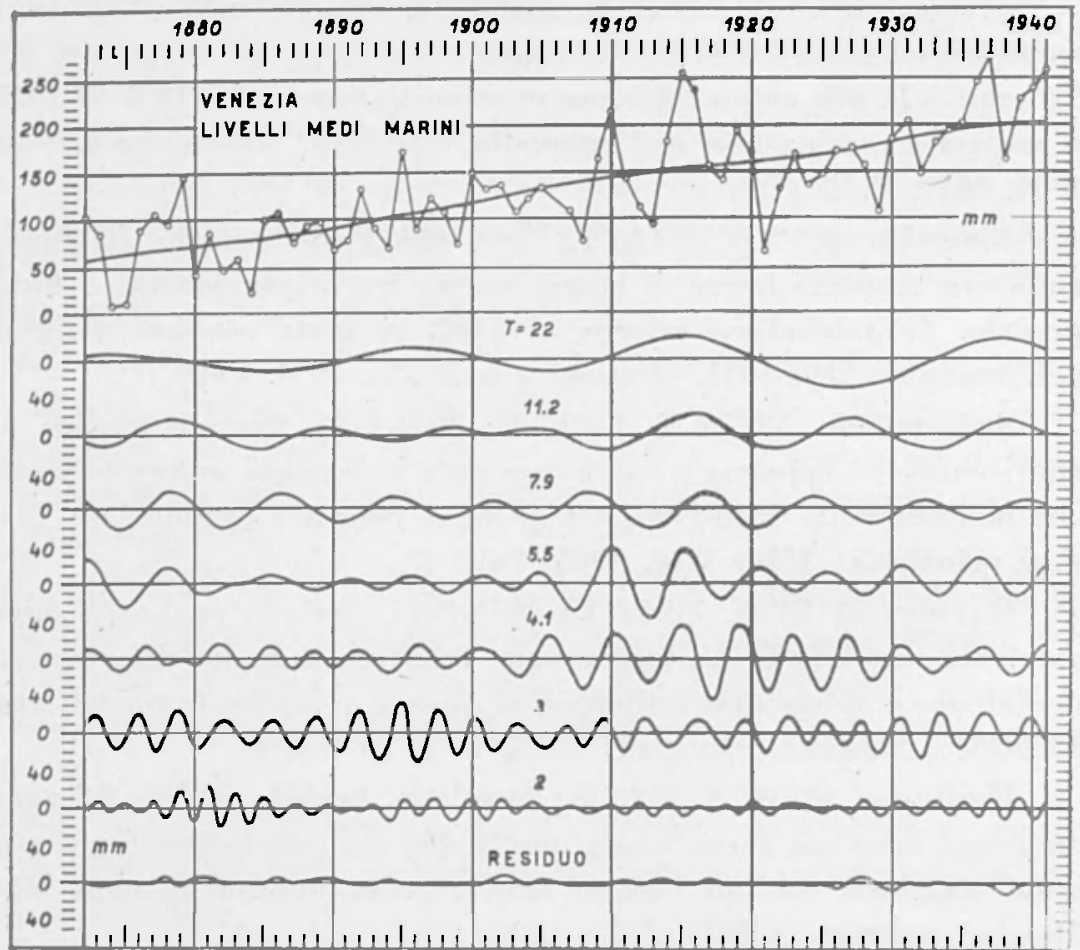

Fig. 3 - Analisi periodale della serie dei livelli marini di Venezia

Bombay. 18 $55^{\prime} \mathrm{N}, 72^{0} 50^{\prime} \mathrm{F}$; 1878-1936. Periodo medio: 7,8 anni; ampiezza massima $2 \mathrm{~cm}$, come l'onda undecennale. Si presenta uniforme con un lieve smorzamento intorno al 1917. Mass. principali: 1894, 1910, 1924. Polli (3).

Rangoon. $18^{\circ} 46^{\prime} \mathrm{N}, 96^{\circ} 10^{\prime} \mathrm{E}$; 1880-1920. Periodo medio: 8 anni; ampiezza massima $3 \mathrm{~cm}$, come quella undecennale. Durante il breve periodo analizzato si presenta bene sviluppata con massimi principali negli anni 1891, 1899. Polli ( $\left.{ }^{3}\right)$.

Port Blair. $11^{0} 41^{\prime}$ N, $92^{\circ} 46^{\prime}$ E; 1880-1920. Periodo medio: 8 anni ; 
ampiezza massima $2 \mathrm{~cm}$, come la componente di 11 anni. Mass. principali : 1901, 1909. Polli $\left({ }^{3}\right)$.

Seattle. $47^{\circ} 36^{\prime} \mathrm{N}, 122^{\circ} 20^{\prime} \mathrm{W}$; 1899-1936. Periodo medio: 8 anni: ampiezza massima $4 \mathrm{~cm}$. Durante il loreve periodo esaminato figura bene sviluppata con massimi principali negli anni 1908, 1925. Polli $\left({ }^{3}\right)$.

San Francisco. $34^{0} 48^{\prime} \mathrm{N}, 1 ? 2^{\prime \prime} 28^{\prime} W^{\prime} ;$ 1898-1936. Periodo medio: 8 anni; ampiezza massima $3 \mathrm{~cm}$, di poco inferiore a quella undecennale. Massimi principali: 1913, 1929. Polli $\left({ }^{3}\right)$.

Buenos Aires. $34^{0} 36^{\prime} \mathrm{S}, 58^{\circ} 22^{\prime} \mathrm{W}$ : 1905-1936. Periodo medio: 8,2 anni; ampiezza massima $9 \mathrm{~cm}$, è la più ampia di tutte le componenti. Massimi principali : 1913, 1921. La serie è troppo breve per poter dedurre altre particolarità. Polli $\left({ }^{3}\right)$.

9. Umidità, evaporazione, nebulosità. - Firenze (Umidità relativa). $43^{0} 47^{\prime} \mathrm{N}, 11^{0} 15^{\prime} \mathrm{E}, \mathrm{h}=75 \mathrm{~m}$; 1856-1918. Periodo medio: 8 anni; ampiezza massima: $4 \%$, figura tra le componenti più sviluppate, presenta uno smorzamento nell'intervallo 1886-1892. Massimi principali : 1871, 1904. Polli $\left({ }^{1}\right)$.

Roma (Umidità relativa). $41^{0} 54^{\prime} \mathrm{N}, 12^{0} 29^{\prime} \mathrm{E}, \mathrm{h}=50 \mathrm{~m}$; 1893 1910. Periodo medio : 8,3 anni; ampiezza massima: $3 \%$, è la maggiore delle componenti aventi periodi superiori agli 5,6 anni. Massimi principali : 1868, 1886. Polli ( $\left.{ }^{11}\right)$.

Firenze (Evaporazione totale annua). Coord. come sopra: 18781918. Periodo medio: 8,3 anni; ampiezza massima: $140 \mathrm{~mm}$, di poco inferiore a quella del ciclo undecennale. Massimi principali: 1892, 1908. Polli $\left({ }^{1}\right)$.

Bucurest (Nebulosità, 0-100 . $44^{0} 25^{\prime} \mathrm{N}, 26^{\circ} 6^{\prime} \mathrm{E}, \mathrm{h}=82 \mathrm{~m}$; 18811930. Periodo medio : 8,3 anni : ampiezza massima 3, circa la metà del ciclo undecennale. Massimi principali: 1894, 1911. Polli ( ${ }^{10}$ ).

Sulina (Nebulosità, 0-100). 45 $09^{\prime} \mathrm{N}, 29^{\circ} 40^{\prime} \mathrm{E}, \mathrm{h}=2 \mathrm{~m}$; 18811930. Periodo medio: 8,1 ; ampiezza massima 6 , è fra le componenti più ampie. Si smorza nel periodo 1900-1910. Massimi principali : 1889, 1915. Polli $\left({ }^{10}\right)$.

10. AcCrescinento degli alberi. - Ravenna. $44^{\circ} 25^{\prime} \mathrm{N}, 12^{0} 15^{\prime} \mathrm{E}$, $\mathrm{h}=5 \mathrm{~m} ;$ 1737-1947. Le analisi periodali degli incrementi meristematici dei pini della zona costiera di Ravenna, eseguite da U. Buli ("), mettono sempre in evidenza una notevole componente di 8 anni. Si presenta generalmente bene sviluppata, con ampiezze variabili che sono 
dell'ordine di quelle della componente undecennale o leggermente inferiori. Massimi principali: 1748, 1794, 1827, 1924.

Ebersualde. $52^{\circ} 50^{\prime} \mathrm{N}, 13^{\circ} 42^{\prime} \mathrm{E}, \mathrm{h}=30 \mathrm{~m} ; 1830-1912$. Alla curva meristenatica degli alseti di questa foresta il Buli ( ${ }^{7}$ ) applicò l'analisi periodale per la ricerca di tutti i cicli componenti. Risulta netta una componente di 8 anni, con ampiezza circa metà di quella del ciclo undecennale. Massimi principali: 1861, 1883.

Dalurne (Svezia centrale); 1820-1910. Serie meristematica degli alseti. L'onda di 8 anni figura chiaramente sino al 1860, poi si smorza per riapparire dal 1875 al 1895 . La sua ampiezza è circa $1 / 3$ di quella della componente undecennale. Massimi principali : 1841, 1885. Buli ( ${ }^{\top}$ ).

Costa norvegese. 1828-1912. Analisi periodale della curva meristematica degli alseti. Periodo medio 8,2 anni; ampiezze leggermente minori di quelle dell'onda undecennale. Massimi principali: 1852, 1874, 1889. Buli $\left({ }^{\top}\right)$.

Danimarca. 1820-1912. Analisi periodale della curva meristematica degli alseti. Periodo medio 8,4 anni: ampiezza di poco inferiore a quella del ciclo undecennale. Massimi principali : $1843,1868,1886$. Buli $\left({ }^{\top}\right)$.

Pilsen. $49^{\circ} 45^{\prime} \mathrm{N}, 13^{\circ} 23^{\prime} \mathrm{E} ; \mathrm{l}=400 \mathrm{~m}$; 1842-1912. Analisi periodale della curva meristematica degli aleti. Periodo medio. 7,8 anni: ampiezza leggermente inferiore a quella dell'onda undecennale. Massimi principali: $1853,1869,1892$. Buli $\left({ }^{7}\right)$.

11. ELENENTI VARI. - Numeri relativi delle macchie solari (numeri di Wolf), 1749-1940. L'analisi periodale di questa serie, eseguita da S. Polli $\left.{ }^{\left({ }^{27}\right.}\right)$, ha messo in evidenza, oltre l'oscillazione di 11,15 anni, una netta e ampia componente avente un periodo medio di 8 anni. Essa ba ampiezza $3 / 4$ di quella undecennale ed è notevolmente superiore a tutte le altre componenti. Presenta massimi sviluppi in corrispondenza a quelli del ciclo di 11 anni, cioè negli intervalli $1770-90$, 1830-50, 1920-50; si indebolisce notevolmente nei periodi 1800-20, 1880 . 1920, in relazione alla minima attività solare. Massimi principali: 1779, 1829, 1871, 1928.

Oscillazione del diametro solare. 1876-1937. Il Cimino $\left({ }^{8}\right)$ mediante l'analisi periodale dei valori annui trova un'oscillazione fondamenmentale con periodo medio di 22,5 anni e una secondaria di 7,5 anni. Il Polli, in una successiva analisi completa degli stessi valori, trova, fra le componenti meglio sviluppate, una con periodo di 7,7 anni. Essa ba un'ampiezza che è dello stesso ordine di quella undecennale 
e $3 / 4$ di quella del ciclo fondamentale di 26 anni. Risulta più intensa nell'intervallo 1915-35. Massimi principali: 1890, 1913, 1929.

Ore di soleggiamento annuo. Trieste, 1886-1939. Periodo medio 7,7 anni. Ampiezza massima 120 ore, circa la metà del ciclo undecennale, che è il più ampio. Si presenta sempre bene sviluppata, cambia fase nell'intervallo 1912-16. Massimi principali: 1894, 1909, 1920, 1935. Polli ( $\left.{ }^{21}\right)$.

Oscillazione del ghiacciaio del Lys (Monte Rosa); 45052' N, 7'48' E, $\mathrm{h}=2300 \mathrm{~m}$ circa; 1789-1931. Nell'analisi periodale della curva delle oscillazioni frontali del ghiacciaio del Lys, eseguita da G. Lovera (16), risulta chiaramente una componente di 8,2 anni con un'ampiezza massima di $40 \mathrm{~m}$. Si smorza e cambia fase nell'intervallo 1865-75, presenta massimi sviluppi negli anni 1810-25, 1845-65, 1880-1900. Massimi principali : 1819, 1855, 1892.

12. Riasstinto dei risultati e conclusioni. - 1) In ciascun elemento considerato, sia terrestre che solare, figura sempre, durante tutto l'intervallo, a meno di qualche breve smorzamento, la fluttuazione avente il periodo medio di 8 anni.

2) Si presenta sempre in tutte le serie dei diversi elementi geofisici di uno stesso sito.

3) Si presenta in tutti i posti della Terra e nei fenomeni solari.

4) $\mathrm{Si}$ presenta sempre con ampiezza notevole e tale che i suoi caratteri sono sempre chiaramente definibili, talvolta risulta la componente più ampia, sorpassa spesso in ampiezza quella undecennale.

5) Il periodo risulta nel 50\% dei casi di anni 8 esatti; nell'11\% di anni 7,9 ; nel $9 \%$ di anni 7,8 : nel $7 \%$ di anni 8,1 ; nel $7 \%$ di anni 8,2 ; due sole volte si eblbero periodi di 7,5 e una sola volta di 8,4 anni. In una stessa analisi varia di poco, al massimo tra 7,5 e 8,5 anni, e ciò avviene quando errori nelle osservazioni o altri fenomeni accidentali alterano il fenomeno naturale.

6) La fase talvolta varia, sia nella stessa analisi, sia tra le diverse regioni. Stessi elementi climatici di una medesima zona la conservano per lunghi intervalli di tempo. Le condizioni di costanza e concordanza di fase non sono dunque completamente soddisfatte. Quando il cambiamento di fase avviene per tutti gli elementi geofisici in un medesimo intervallo di tempo, come ad es. si osserva nel periodo 1880-90, la causa ha carattere generale, trascende il soggetto, e conferma la realtà dell'onda; se il cambiamento di fase è solamente locale e limitato ad un solo elemento, e ciò avviene molto raramente 
(nel caso presente 2 volte su 50 ), la causa è insita nella località ed è da ricercarsi in cambiamenti subentrati nelle osservazioni o in errori sistematici commessi prima o a partire da quell'epoca. Lo stesso ciclo undecennale presenta nei diversi elementi geofisici e nelle diverse regioni della Terra, sfasamenti molto ampi, per non dire opposizioni.

7) Presenta particolari caratteri osservabili nella generalità degli elementi e dei siti: notevole sviluppo nell'intervallo 1830-50, irregolarità, smorzamenti e cambiamenti di fase nel periodo 1885-95.

I'oscillazione climatica di 8 anni soddisfa dunque a tutte le condizioni necessarie e sufficienti per poterla ritenere un ciclo climatico avente realtà fisica. Risulta uno dei cicli meglio sviluppati, presenta ampiezza dell'ordine di quella del ciclo undecennale, spesso anzi le supera. Determina un'effettiva fluttuazione climatica che estrapolata contribuirà con notevole peso, assieme alle altre componenti fondamentali, alla sintesi dell'andamento climatico futuro.

$$
\text { Trieste - Istituto Talassografico - Novembre } 1949 .
$$

\section{RIASSUNTO}

Premesse le condizioni perché un ciclo climatico, in qualsiasi maniera determinato, possa considerarsi una realtà fisica ed esaminate oltre 60 analisi periodali, armoniche e varie di lunghe serie di elementi climatici, si mette in evidenza l'esistenza, la notevole ampiezza e la realtà fisica dol ciclo di 8 anni. Esso si presenta sempre, in tutti gli elementi geofisici $e$ in tutte le regioni della Terra. $t$ ampio come quelli di 11 e 5,6 anni, mantiene costante il periodo, la fase varia con la regione; presenta particolari amplificazioni e smorzamenti di carattere universale.

\section{BIBLIOGRAFIA}

(1) Alfani G.: Un secolo di osserv, meteor. Pubbl. 126, Osserv. Ximen., Firenze, 1920.

(2) Alt E.: Klimakunde von Mittel-u. Südeuropa. Borntraeger, Berlin, 1932.

(3) Ass. Ocfan. Phys.: Monthly and annual mean heights of sea-level. Publ. Sc. 5, Liverpool, 1940.

(4) Baur F.: Das Periodogramm 100 jähriger Temperaturbeobach. in Berlin. Met. Zeitschr., 1927, 11, 414-418. 
(5) Bramanti L.: Analisi di una serie pluviometrica. Geofisica pura e appl., voI. VIII, 5-6, 1946.

(6) BruckNer E.: Klimaschwankungen seit 1700 nebst Bemerkungen über... Geogr. Abhandl., Wien, Bd. IV, H. 2, 1890.

(7) Bulı P.: Ricerche climatiche sulle pinete di Ravenna. Cons. Naz. Ric, Centro Studi Geogr. Fis.; II. Variaz. clima it., n. 10, Bologna, 1949.

(8) Cimino M.: Un'oscillazione ventiduennale nel diametro solare. Pontif. Acad. Scient., Comment., voI. VIlI, n. 17, 1944.

(9) Davis. G. G.: Clima de la Rep. Argentina. Buenos Aires, 1902.

(10) Dissescu C.: Variatia nebulositatii in Romania. Inst. Met. Centr., Mem. II, $1,1933$.

(11) Eredia F.: Il clima di Roma. Annali Uff. Centr. Meteor, vol. 32, 1910.

(12) Hlavac V.: Die 165 jährige Prager Temperaturreihe 1775 bis 1939. Met. Zeits, Juli, 1940.

(13) Кмоск K.: Klimakunde von Südamerika. Borntraeger, Berlin, 1930.

(14) LaBRIJN A.: The climate of the Netherlands during the last two and half centuries. Meded. en Verhand. n. 49. Kon. Nederl. Meteorologisch Inst. n. 102; 1945.

(15) Lovera G.: Sulle temperature medie mensili e annue a Torino. Atti Acc. Scienze, voI. 78, Torino 1942-43.

(16) - Analisi periodale delle oscillazioni del ghiacciaio del Lys e dell'anda. mento di alcuni elementi meteorologici connessi. Boll. Comit. Glaciol. Ital., n. 25, 1948.

(17) Marvin C. F.: A new principle in the Analysis of Periodicities. Monthly Weather Review, 1924.

(18) MaURER J.: Die periodische Wiederkehr hohen Luftdruckstandes im W'inter des Alpengebiets. Meteor. Zeitschr., 1918, 3-4, p. 95-98.

(19) Moore H. L.: Economic cycles: their law and cause. New York, The Macmillan Co., 1914.

(20) NeAcler W.: Statistik der Temperaturverhältnisse von Leipzig (1831-1935). Berichten der Math.Phys. Kl. der Sachs. Ak. der Wiss., Leipzig. LXXXVIII Bd. (18 maggio 1936).

(21) Pollı S.: Cinquanta anni di misure eliofanometriche a Trieste. Riv. di Meteor. I, 24 N. S., 1939.

(22) - Analisi periodale di una serie pluviometrica bisecolare. Padova 1727. 1940. Riv. di Meteor. Aeron., I, 1943.

(23) - Analisi periodale di due serie climatiche centennali, Trieste 1841-1940. Arch. di Ocean. e Limn., fasc. 2-3, Mem. 297, Com. Talass. It., 1942.

(24) - Analisi periodale di tre serie di livelli medi marini, Harlingen, Massluis, Vlissingen. Arch. di Ocean. e Limn. A. II, 1.2, Mem. 302, Com. Talass., 1943.

(25) - Analisi periodale della serie pluviometrica di Mantova. Geof. pura e appl., voI. VII, 14, 1945.

(20) - La realià fisica del ciclo climatico di 5,6 anni. Geof. pura e appl., VIII, 34, 94-103; 1946.

(27) - Analisi periodale dei numeri relativi delle macchie solari. Geof. pura e appl., IX, 3.6, 95.105; 1946.

(28) - Analisi periodale delle serie dei livelli marini di Trieste e Venezia. Geof. pura e appl., X, 1-2, 29-40; 1947. 
(-9) StumpfF K.: Grundlagen und Methoden der Periodenforschung. Borntraeger, Berlin 1937.

(30) - Ermittlung und Realität von Periodizitäten. Borntraeger, Berlin 1940.

(31) Uff. Idrog. Magistr. Acqve: Boll. Mens. e Annali Idrologici.

(32) Vercelli F.: L'Aria. U.T.E.T, Torino 1933.

(33) - Grida per l'analisi delle periodicità nei diagrammi oscillanti. Ment. 28.5, Comit. Talassogr. It., C.N.R., Roma, 1940.

(34) Wagner A.: Eine bemerkenswerte 16 jährige Klimaschwankung. Sitzbr. Ak. Wiss. Wien, Mat. Nat. Kl. 133, 1924.

(35) WoeIKof A.: Perioden in der Temperatur von Stocklolm. Meteor. Zeitschr, Bd. 23, 10. 433.436; 1906. 